\title{
QUARTIC K3 SURFACES WITHOUT NONTRIVIAL AUTOMORPHISMS
}

\author{
RONALD VAN LUIJK
}

\begin{abstract}
For any field $k$ of characteristic at most 19 we exhibit an explicit smooth quartic surface in $\mathbb{P}_{k}^{3}$ with trivial automorphism group over $\bar{k}$. We also show how this can be extended to higher characteristics. Over $\mathbb{Q}$ we construct an example on which the set of rational points is Zariski dense.
\end{abstract}

\section{Introduction}

For any field $k$ we fix an algebraic closure of $k$, denoted by $\bar{k}$. For a variety $X \subset \mathbb{P}_{k}^{n+1}$ we set $\bar{X}=X \times_{k} \bar{k}$, we say that $X$ is smooth if $\bar{X}$ is regular, and we let Aut $\bar{X}$ denote the group of $\bar{k}$-automorphisms of $\bar{X}$, while Lin $\bar{X}$ denotes the group of linear automorphisms of $\bar{X}$, i.e., automorphisms induced by a linear transformation of the coordinates of $\mathbb{P}^{n+1}$. The following theorem was proved by Poonen, see [10], Thm. 1.6.

Theorem 1.1. For any field $k$ and integers $n \geq 1, d \geq 3$ with $(n, d) \neq(1,3)$, there exists a smooth hypersurface $X$ in $\mathbb{P}_{k}^{n+1}$ of degree $d$ with $\operatorname{Lin} \bar{X}=\{1\}$.

Remark 1.2. For $(n, d)=(1,3)$ no such hypersurface exists. In that case we get a plane cubic curve. With a flex as the origin, such a curve obtains the structure of an elliptic curve on which multiplication by -1 is a nontrivial linear automorphism.

Poonen's proof of Theorem 1.1 consists of giving carefully crafted explicit examples. For fields that are large enough, a nonconstructive proof can be deduced from various older results. For details we refer to [10] and the references given there. The next theorem states that in many cases all automorphisms are linear, see [2], Thm. 1, for dimension 1, and [8], Thm. 2, for higher dimension.

Theorem 1.3. If $X$ is a smooth hypersurface in $\mathbb{P}^{n+1}$ of degree $d$ with $n \geq 1, d \geq 3$, and $(n, d) \notin\{(1,3),(2,4)\}$, then we have Aut $\bar{X}=\operatorname{Lin} \bar{X}$.

Combining Theorem 1.1 and 1.3 we find the following corollary.

Corollary 1.4. For any field $k$ and integers $n \geq 1, d \geq 3$ with $(n, d) \notin\{(1,3),(2,4)\}$, there exists a smooth hypersurface $X$ in $\mathbb{P}_{k}^{n+1}$ of degree $d$ with Aut $\bar{X}=\{1\}$.

In this paper we deal with the remaining case $(n, d)=(2,4)$ in small characteristics, including zero. The following theorem states our main result.

Theorem 1.5. Let $k$ be any field of characteristic at most 19. Then there exists a smooth quartic surface $X \subset \mathbb{P}_{k}^{3}$ with Aut $\bar{X}=\{1\}$.

Received by the editors December 18, 2005. 
Smooth quartic surfaces in $\mathbb{P}^{3}$ are examples of K3 surfaces. Some do have nonlinear automorphisms. The essential difference with the hypersurfaces in Theorem 1.3 is that the canonical sheaf is trivial and the Picard group Pic $\bar{X}$ may be larger than $\mathbb{Z}$. The arithmetic of K3 surfaces is not well understood in general. It is for instance not known whether there exists a K3 surface over a number field on which the set of rational points is neither empty nor dense. Bogomolov and Tschinkel [1] proved that if a K3 surface $X$ over a number field $K$ has an infinite automorphism group or it admits an elliptic fibration (not necessarily with a section), then there exists a finite field extension $L$ of $K$, such that the set $X(L)$ of $L$-rational points on $X$ is Zariski dense. The next theorem shows that it is not always necessary to extend $K$, even if the automorphism group is trivial and we have $K=\mathbb{Q}$.

Theorem 1.6. Let $X \subset \mathbb{P}_{\mathbb{Q}}^{3}(x, y, z, w)$ be the surface given by

$$
\begin{aligned}
2 x^{3} w+2 x^{2} z^{2} & +2 x^{2} z w+x^{2} w^{2}+2 x y^{2} w+6 x y z^{2}+2 x y w^{2}+x z^{2} w+ \\
& +2 y^{3} z+6 y^{2} z^{2}+y^{2} w^{2}+10 y z^{2} w+z^{3} w+7 z^{2} w^{2}-4 z w^{3}=0 .
\end{aligned}
$$

Then $X$ is smooth, we have Aut $\bar{X}=\{1\}$, and the set $X(\mathbb{Q})$ is Zariski dense in $X$.

The surface in Theorem 1.6 admits an elliptic fibration. If the result of Bogomolov and Tschinkel does not apply, then there may still be infinitely many rational points, as shown by the next theorem.

Theorem 1.7. Let $X \subset \mathbb{P}_{\mathbb{Q}}^{3}(x, y, z, w)$ be the surface given by $w\left(3 x^{3}+3 x y^{2}-x y w+3 x z w-x w^{2}+y^{3}-y^{2} w+2 z^{3}+w^{3}\right)=(x y+x z+y z)(x y+x z+3 y z)$.

Then $X$ is smooth and does not admit an elliptic fibration over $\overline{\mathbb{Q}}$. We have Aut $\bar{X}=$ $\{1\}$ and the set $X(\mathbb{Q})$ is infinite.

To prove Theorem 1.5 we will write down explicit examples of quartic surfaces $X$ and show that we have both $\operatorname{Aut} \bar{X}=\operatorname{Lin} \bar{X}$ and $\operatorname{Lin} \bar{X}=\{1\}$. This is done in section 3 and in a different way in section 4 . The main idea behind both these sections is described in section 2. As in [10], it is not particularly hard to check that our examples have no nontrivial linear automorphisms. The hard part was to find examples for which it is doable to verify this by hand. In section 5 we prove Theorems 1.5, 1.6, and 1.7.

Our method works for any characteristic. We are limited, however, by the lack of the ability to compute the characteristic polynomial of Frobenius acting on certain cohomology groups in large characteristics. This is used to show $\operatorname{Aut} \bar{X}=\operatorname{Lin} \bar{X}$.

\section{The idea}

The following lemma is the key ingredient to proving $\operatorname{Aut} \bar{X}=\operatorname{Lin} \bar{X}$.

Lemma 2.1. Let $X$ be a normal complete intersection in $\mathbb{P}^{n}$ and let $\sigma$ be a $\bar{k}$ automorphism of $\bar{X}$. If $\sigma$ sends a hyperplane section of $\bar{X}$ to another hyperplane section of $\bar{X}$, then we have $\sigma \in \operatorname{Lin} \bar{X}$.

Proof. Since $\sigma$ sends a hyperplane section to a hyperplane section, it fixes $\mathcal{O}_{X}(1)$, so it sends a basis of $H^{0}\left(X, \mathcal{O}_{X}(1)\right)$ to another basis. By [5], Exc. II.8.4.c, the map $H^{0}\left(\mathbb{P}^{n}, \mathcal{O}_{\mathbb{P}^{n}}(1)\right) \rightarrow H^{0}\left(X, \mathcal{O}_{X}(1)\right)$ is surjective, so the change of basis of $H^{0}\left(X, \mathcal{O}_{X}(1)\right)$ 
is induced by a change of basis of $H^{0}\left(\mathbb{P}^{n}, \mathcal{O}_{\mathbb{P}^{n}}(1)\right)$. The lemma now follows from [5], Thm. II.7.1.

For the remaining of this paper we will assume that $X$ is a smooth quartic surface in $\mathbb{P}^{3}$. The condition of Lemma 2.1 is equivalent to $\sigma$ fixing the class of hyperplane sections in the Picard group $\operatorname{Pic} \bar{X}$. This is the group of divisor classes on $\bar{X}$ modulo linear equivalence. The Néron-Severi group $\mathrm{NS}(\bar{X})$ is the group of divisor classes modulo algebraic equivalence. For a precise definition of algebraic equivalence, see [5], Exc. V.1.7. The group $\operatorname{NS}(\bar{X})$ is a finitely generated group. Its rank $\rho=$ $\operatorname{dim}_{\mathbb{Q}} \mathrm{NS}(\bar{X}) \otimes \mathbb{Q}$ is called the Picard number of $X$. Note that in other papers $\rho$ is sometimes called the geometric Picard number of $X$.

For K3 surfaces, in particular for smooth quartic surfaces in $\mathbb{P}^{3}$, linear equivalence is the same as algebraic and numerical equivalence. This means that two divisors on $\bar{X}$ are linearly equivalent if and only if they have the same intersection number with all other divisors. It implies that $\operatorname{Pic} \bar{X}$ is finitely generated and free, isomorphic to $\operatorname{NS}(\bar{X})$ and the intersection pairing endows $\operatorname{Pic} \bar{X}$ with the structure of a lattice. For a divisor $D$, let $[D]$ denote the divisor class in $\operatorname{Pic} \bar{X} \cong \mathrm{NS}(\bar{X})$. We will say that a divisor $D$ on $\bar{X}$ is very ample if there exists an integer $m$ and a closed immersion $\varphi: \bar{X} \rightarrow \mathbb{P}^{m}$ such that $\varphi(D)$ is a hyperplane section on $\varphi(\bar{X})$.

Lemma 2.2. Let $X$ be a smooth quartic surface in $\mathbb{P}^{3}$ and $C$ a smooth, geometrically irreducible curve on $X$. Then we have $C^{2}=2 g-2$, where $g$ denotes the genus of $C$.

Proof. Since the canonical divisor on $X$ is trivial (see [5], Exm. II.8.20.3), this follows from the adjunction formula, see [5], Prop. V.1.5.

Proposition 2.3. Let $X$ be a smooth quartic surface in $\mathbb{P}^{3}$ and let $\sigma$ be a $\bar{k}$-automorphism of $\bar{X}$. Let $H$ denote the divisor class of hyperplane sections. Then the following implications hold.

(1) If $\mathrm{NS}(\bar{X})$ is generated by $H$ then we have $\sigma \in \operatorname{Lin} \bar{X}$.

(2) If $\mathrm{NS}(\bar{X})$ is generated by $H$ and the divisor class of a line $L$, then we have $\sigma \in \operatorname{Lin} \bar{X}$ and $\sigma$ fixes $L$.

(3) If $\mathrm{NS}(\bar{X})$ is generated by $H$ and the divisor class of a conic $C$, then we have $\sigma \in \operatorname{Lin} \bar{X}$ and $\sigma$ fixes the plane that contains $C$.

Proof. Without loss of generality we may assume $\bar{X}=X$. Since the induced automorphism $\sigma_{*}$ of $\operatorname{NS}(\bar{X})$ preserves intersection numbers, it is an automorphism of $\mathrm{NS}(\bar{X})$ as a lattice. Set $H^{\prime}=\sigma_{*}(H)$. Note that $\sigma_{*}$ sends very ample divisor classes to very ample divisor classes and effective divisor classes to effective divisor classes, so $H^{\prime}$ is a very ample divisor class.

Case (1). The divisor classes $H^{\prime}$ and $H$ both generate $\mathrm{NS}(\bar{X}) \cong \mathbb{Z}$, so we have either $H^{\prime}=-H$ or $H^{\prime}=H$. As $H^{\prime}$ is effective, we find $H^{\prime}=H$ and by Lemma 2.1 we have $\sigma \in \operatorname{Lin} \bar{X}$.

Case (2). We have $H^{2}=\operatorname{deg} X=4$ and $H \cdot[L]=\operatorname{deg} L=1$, see [5], Exc. V.1.2. By Lemma 2.2 we have $L^{2}=-2$. This means that with respect to the basis $\{H,[L]\}$ the lattice $\mathrm{NS}(\bar{X})$ has Gram matrix

$$
\left(\begin{array}{cc}
4 & 1 \\
1 & -2
\end{array}\right)
$$


and thus discriminant -9 . The automorphism group of this lattice is isomorphic to $(\mathbb{Z} / 2 \mathbb{Z})^{2}$ and it is generated by the automorphisms $[-1]: x \mapsto-x$ and $\tau: H \mapsto$ $H+[L],[L] \mapsto-[L]$. The only automorphism that sends effective divisor classes to effective divisor classes is the identity, so we find $\sigma_{*}=\mathrm{Id}$. This implies that every hyperplane section is sent to a divisor that is linearly equivalent to it, i.e., another hyperplane section. From Lemma 2.1 we get $\sigma \in \operatorname{Lin} \bar{X}$. We also conclude that $\sigma$ maps $L$ to an effective divisor $L^{\prime}$ that is linearly equivalent to $L$. As two different irreducible effective divisors have a nonnegative intersection number, we conclude from $L^{\prime} \cdot L=L^{2}=-2$ that we have $L^{\prime}=L$, so $\sigma$ fixes $L$.

Case (3). We find similarly to case (2) that with respect to the basis $\{H,[C]\}$ the lattice $\mathrm{NS}(\bar{X})$ has Gram matrix

$$
\left(\begin{array}{cc}
4 & 2 \\
2 & -2
\end{array}\right)
$$

and thus discriminant -12 . Note that the conic $C$ is contained in a plane $V$, see [5], Exc. IV.3.4. The other component $\tilde{C}$ in $V \cap \bar{X}$ has degree $\operatorname{deg} \bar{X} \cdot \operatorname{deg} V-\operatorname{deg} C=2$, so it is also a conic, which a priori might be degenerated. Let $a$ and $b$ be integers such that $H^{\prime}=a H+b[C]$. Since $H^{\prime}$ is very ample, it has positive intersection number with the effective divisor classes $[C]$ and $[\tilde{C}]=H-[C]$ (see [5], Thm. V.1.10), so we find

$$
\begin{aligned}
& 0<H^{\prime} \cdot[C]=(a H+b[C]) \cdot[C]=2 a-2 b \\
& 0<H^{\prime} \cdot[\tilde{C}]=(a H+b[C]) \cdot(H-[C])=2 a+4 b
\end{aligned}
$$

We have $4=H^{2}=H^{\prime 2}=4 a^{2}+4 a b-2 b^{2}$, so we deduce $4=(2 a-2 b)(2 a+4 b)+6 b^{2}>$ $6 b^{2}$, which implies $b=0$. From $H^{\prime 2}=4$ and the inequalities above we conclude $a=1$, so $\sigma_{*} H=H^{\prime}=H$. Again by Lemma 2.1 we have $\sigma \in \operatorname{Lin} \bar{X}$. The orthocomplement of $H$ in $\mathrm{NS}(\bar{X})$ is generated by $D=2[C]-H$, so we find $\sigma_{*} D= \pm D$. This implies $\sigma_{*}[C]=[C]$ or $\sigma_{*}[C]=H-[C]=[\tilde{C}]$. This means that $\sigma$ sends $C$ to a divisor that is linearly equivalent to $C$ or to $\tilde{C}$. As both $C$ and $\tilde{C}$ have negative self intersection, this implies as in case (2) that $\sigma$ maps $C$ to $C$ or to $\tilde{C}$. Since $V$ is the unique plane containing $C$ or $\tilde{C}$, the automorphism $\sigma$ fixes $V$.

The following lemma will be useful in conjunction with Proposition 2.3.

Lemma 2.4. Let $X$ be a smooth quartic surface in $\mathbb{P}^{3}$ and let $H$ denote the class of hyperplane sections. Let $\rho$ denote the Picard number of $X$. Then the following implications hold.

(1) If $\rho \leq 1$, then $\mathrm{NS}(\bar{X})$ is generated by $H$.

(2) If $\rho \leq 2$ and $\bar{X}$ contains a line $L$, then $\mathrm{NS}(\bar{X})=\langle H,[L]\rangle$.

(3) If $\rho \leq 2$ and $\bar{X}$ contains a conic $C$, then $\operatorname{NS}(\bar{X})=\langle H,[C]\rangle$.

Proof. Without loss of generality we may assume that the ground field is algebraically closed, so we have $X=\bar{X}$. We say that a lattice $\Lambda$ with its pairing given by $(x, y) \mapsto$ $x \cdot y$ is even if the norm $x \cdot x$ is even for all $x \in \Lambda$. From Lemma 2.2 we find that the integral lattice $\mathrm{NS}(X)$ is generated by elements of even norm, so $\mathrm{NS}(X)$ is an even lattice. Note that the discriminants of a lattice $\Lambda$ and a sublattice $\Lambda^{\prime}$ of finite index in $\Lambda$ are related by $\operatorname{disc} \Lambda^{\prime}=\left[\Lambda: \Lambda^{\prime}\right]^{2} \cdot \operatorname{disc} \Lambda$. 
Case (1). From $H^{2}=4$ we find $H \neq 0$, so $\rho=1$ and the lattice $\langle H\rangle$ has finite index in $\operatorname{NS}(X)$. This implies $[\mathrm{NS}(X):\langle H\rangle]^{2} \cdot \operatorname{disc} \mathrm{NS}(X)=\operatorname{disc}\langle H\rangle=H^{2}=4$. As any 1-dimensional even lattice has even discriminant, we find that disc $\operatorname{NS}(X)$ is even, so $[\mathrm{NS}(X):\langle H\rangle]=1$, and $\mathrm{NS}(X)$ is generated by $H$.

Case (2). As in the proof of Proposition 2.3, the lattice $\langle H,[L]\rangle$ is 2-dimensional and has discriminant -9 . Therefore we have $\rho=2$ and $[\mathrm{NS}(X):\langle H,[L]\rangle]^{2} \cdot \operatorname{disc} \mathrm{NS}=-9$. We conclude $\operatorname{NS}(X)=\langle H,[L]\rangle$ or $\operatorname{disc} \operatorname{NS}(X)=-1$. By the classification of even unimodular lattices, the latter case implies that $\mathrm{NS}(X)$ is isomorphic to the lattice with Gram matrix

$$
\left(\begin{array}{ll}
0 & 1 \\
1 & 0
\end{array}\right)
$$

which is impossible for a quartic surface by a theorem of Van Geemen, see [13], 5.4. We find that $\mathrm{NS}(X)$ is indeed generated by $H$ and $[L]$.

Case (3). As in the proof of Proposition 2.3, the lattice $\langle H,[C]\rangle$ is 2-dimensional with discriminant -12 and Gram matrix

$$
\left(\begin{array}{cc}
4 & 2 \\
2 & -2
\end{array}\right)
$$

Therefore we have $[\mathrm{NS}(X):\langle H,[C]\rangle]^{2} \cdot \operatorname{disc} \mathrm{NS}=-12$. We conclude that the index $[\mathrm{NS}(X):\langle H,[C]\rangle]$ divides 2. Take $D \in \mathrm{NS}(X)$. Then we have $2 D=a H+b[C]$ for some integers $a, b$. Since $\operatorname{NS}(X)$ is even, we find $8 \mid 4 D^{2}=(A H+b[C])^{2}=4 a^{2}+4 a b-$ $2 b^{2}$. This implies that $a$ and $b$ are both even, so we have $D \in\langle H,[C]\rangle$. Since this holds for all $D \in \mathrm{NS}(X)$, we find $\operatorname{NS}(X)=\langle H,[C]\rangle$.

In view of implication (1) of Proposition 2.3 and Lemma 2.4, one approach to proving Theorem 1.5 is to take Poonen's examples of quartic surfaces $X$ with $\operatorname{Lin} \bar{X}=$ $\{1\}$ (see [10]) and prove that one of them has Picard number 1. There are at least two problems with this approach. First of all, by Tate's conjecture (see [12]) the NéronSeveri group of a smooth quartic surface in $\mathbb{P}^{3}$ over a field of positive characteristic has even rank. As Tate's conjecture has been proved for all smooth quartic surfaces in characteristic $p \geq 5$ that are ordinary (see [9]), there is not much hope for this approach in positive characteristic. The second problem lies in proving that the Picard number of an explicit quartic surface $\bar{X}$ over a field of characteristic 0 equals 1. The only way known to do this is described in [15]. It requires two primes of good reduction for which we know the discriminant of the Néron-Severi lattice of the reduction up to a square factor. It is not clear how to obtain this.

Another way to use (1) of Proposition 2.3 is to take the quartic surfaces $X$ with Picard number 1 that the author found in [15] and prove that they satisfy $\operatorname{Lin} \bar{X}=\{1\}$. The problem with this approach is that these surfaces are defined by fairly erratic polynomials, which makes it hard to get a handle on their linear automorphisms.

We will therefore use only implications (2) and (3) of Proposition 2.3. This gives the extra advantage of knowing a specific subvariety that is fixed under any automorphism $\sigma$, which severely restricts the possibilities for such $\sigma$.

Let $X$ be a smooth surface over a number field $K$ and let $\mathfrak{p}$ be a prime of good reduction with residue field $k$. Let $\mathfrak{X}$ be an integral model for $X$ over the localization $\mathcal{O}_{\mathfrak{p}}$ of the ring of integers $\mathcal{O}$ of $K$ at $\mathfrak{p}$. Let $l$ be any extension field of $k$. Then by 
abuse of notation we will write $X \times l$ for $\mathfrak{X} \times{ }_{\operatorname{Spec}} \mathcal{O}_{\mathfrak{p}}$ Spec $l$. To bound the Picard number of $X$ we will use the following propositions.

Proposition 2.5. Let $X$ be a smooth surface over a number field $K$ and let $\mathfrak{p}$ be a prime of good reduction with residue field $k$. Then we have

$$
\operatorname{rank} \mathrm{NS}(X \times \bar{K}) \leq \operatorname{rank} \mathrm{NS}(X \times \bar{k}) .
$$

Proof. See [4], Example 20.3.6. It also follows from a natural injection $\operatorname{NS}(X \times \bar{K}) \otimes$ $\mathbb{Q} \hookrightarrow \mathrm{NS}(X \times \bar{k}) \otimes \mathbb{Q}$, see [14], Prop. 6.2.

Proposition 2.6. Let $X$ be a smooth surface over a finite field $k$ with $q$ elements. Let $l$ be a prime not dividing $q$. Let $F$ denote the automorphism on $H_{\text {ét }}^{2}\left(\bar{X}, \mathbb{Q}_{l}\right)(1)$ induced by $q$-th power Frobenius. Let $t$ denote the number of eigenvalues of $F$ that are roots of unity, counted with multiplicity. Then there is a natural injection

$$
\mathrm{NS}(\bar{X}) \otimes \mathbb{Q}_{l} \hookrightarrow H_{\text {ét }}^{2}\left(\bar{X}, \mathbb{Q}_{l}\right)(1)
$$

that respects the action of Frobenius and we have $\operatorname{rank} \mathrm{NS}(\bar{X}) \leq t$.

Proof. See [14], Prop. 6.2 and Cor. 6.4. Note that in the referred corollary Frobenius acts on the cohomology group $H_{\text {ét }}^{2}\left(\bar{X}, \mathbb{Q}_{l}\right)$ without a twist. Therefore, the eigenvalues are scaled by a factor $q$.

Remark 2.7. Tate's conjecture (see [12]) states that the inequality in Proposition 2.6 is in fact an equality.

One way to find the characteristic polynomial of Frobenius is through the Lefschetz formula. For a smooth, projective variety $X$ over the field $\mathbb{F}_{q}$ and a positive integer $n$, this formula relates the number of points on $X$ over $\mathbb{F}_{q^{n}}$ to the traces of $F_{i}^{n}$ for $0 \leq i \leq 4$, where $F_{i}$ is the $q$-th power Frobenius acting on $H_{\text {êt }}^{i}\left(\bar{X}, \mathbb{Q}_{l}\right)$. For a K3 surface only the action for $i=2$ is unknown, so the trace of $F_{2}^{n}$ can be computed from $\# X\left(\mathbb{F}_{q^{n}}\right)$. From these traces for various $n$ one can deduce the characteristic polynomial of $F_{2}$. If a priori a Galois invariant subspace $V$ of $H_{\text {ét }}^{2}\left(\bar{X}, \mathbb{Q}_{l}\right)$ is known, then it suffices to find the characteristic polynomial of Frobenius on the quotient, which requires fewer traces. In our case $V$ will always be generated by the class of hyperplanes and the class of either a line or a conic. For details see [14], section 6 and 7, and [15], section 2. A faster way to find the number $t$ as in Proposition 2.6 is to use Kedlaya's algorithm based on De Rham cohomology [7], analogous to his algorithm for hyperelliptic curves described in [6]. We will use both methods freely without reproducing the details that can be found in these references.

The following lemmas will be used in sections 3 and 4 .

Lemma 2.8. Let $Z$ be a smooth, irreducible hypersurface in $\mathbb{P}^{n}$ over an algebraically closed field, with $n \geq 3$. Let $H$ be a hyperplane not equal to $Z$ and let $C$ be an irreducible component of the scheme theoretic intersection $H \cap Z$. Then $C$ is reduced and the intersection multiplicity of $H$ and $Z$ along $C$ is equal to 1.

Proof. Let $S=k\left[x_{0}, \ldots, x_{n}\right]$ denote the homogeneous coordinate ring of $\mathbb{P}^{n}$. Suppose $Z$ is given by $f=0$ for some $f \in S$. Without loss of generality we may assume that $H$ is given by $x_{n}=0$. Then $H$ is isomorphic to $\mathbb{P}^{n-1}$ with homogeneous coordinate ring $T=k\left[x_{0}, \ldots, x_{n-1}\right]$ and $H \cap Z$ is given by the image $g$ of $f$ under the 
homomorphism $S \rightarrow T$ that sends $x_{n}$ to 0 . Since $T$ is a unique factorization domain, the component $C$ corresponds to an irreducible factor $h$ of $g$. We find from the definition of intersection multiplicity (see [5], p. 53) that the intersection multiplicity of $H$ and $Z$ along $C$ is equal to 1 if and only if the exponent of $h$ in $g$ equals 1 , which is the case if and only if the component $C$ is reduced. Suppose the exponent of $h$ in $g$ were at least 2 . Then we could write $f=x_{n} q+h^{2} p$ for some $q \in S$ and $p \in R$. This implies that for every point on $C$, which is given by $x_{n}=h=0$, we have $f=0$ and $\partial f / \partial x_{i}=0$ for $i \in\{0, \ldots, n-1\}$. From $n \geq 3$ we conclude that $C$ has dimension at least 1. From the Projective Dimension Theorem (see [5], Thm. I.7.2) it follows that there is a point $P$ on $C$ that also satisfies $\partial f / \partial x_{n}=0$, which implies that $Z$ is singular at $P$. The lemma follows from this contradiction.

Lemma 2.9. Let $k$ be a field of characteristic different from 2 and 3 . Let $X$ be a K3 surface over $k$ with Picard number at most 2 . Let $\pi: X \rightarrow \mathbb{P}^{1}$ be an elliptic fibration. Then all singular fibers are irreducible curves with either a node or a cusp. Let $n$ and $c$ denote the number of nodal and cuspidal fibers respectively. Then we have $n+2 c=24$.

Proof. Let $F$ be a fiber of $\pi$ with $r$ irreducible components. By [11], Prop. III.8.2, these components generate a sublattice of $\mathrm{NS}(\bar{X})$ of rank $r$ in which every element $z$ satisfies $z^{2} \leq 0$. Any ample divisor has positive self intersection and therefore none of its multiples is contained in this sublattice, so the Picard number of $\bar{X}$ is at least $r+1$. This proves that all fibers are irreducible. In general, all irreducible singular fibers of an elliptic fibration are nodal or cuspidal curves. For any fiber $F$, let $e(F)$ denote the Euler characteristic of $F$. For K3 surfaces, we have $\sum_{F} e(F)=24$, where the sum is taken over all singular fibers $F$ of $\pi$, see [3], Cor. 7.16 and p. 178. The lemma follows from the fact that outside characteristic 2 and 3, nodal and cuspidal fibers have Euler characteristic 1 and 2 respectively.

Lemma 2.10. Let $f \in \mathbb{R}[x]$ be a polynomial of degree $d$ all of whose roots have complex absolute value equal to 1 . Then we have $x^{d} f\left(x^{-1}\right)=(-1)^{n} f(x)$, where $n$ is the order of vanishing of $f$ at $x=1$.

Proof. The only real numbers of absolute value 1 are \pm 1 , so the factorization of $f$ into irreducible factors over $\mathbb{R}$ is

$$
f=c(x-1)^{n}(x+1)^{m} \prod_{i=1}^{r} g_{i},
$$

for some constant $c$, integers $m, n, r$, and quadratic monic polynomials $g_{i}$. Since the conjugate roots of $g_{i}$ have absolute value 1 , we have $g_{i}=x^{2}+a_{i} x+1$ for some $a_{i} \in \mathbb{R}$, so $x^{2} g_{i}\left(x^{-1}\right)=g_{i}(x)$. The lemma follows.

\section{Explicit examples containing a line}

Proposition 3.1. Let $k$ be any field and let $f_{1}, f_{2}, f_{3} \in k[x, y, z, w]$ be homogeneous polynomials of degree 2 . Let $X$ be the surface in $\mathbb{P}^{3}$ given by

$$
w\left(x^{3}+x y^{2}+w f_{1}\right)+z\left(y^{3}+z f_{2}\right)+z w f_{3}=0 .
$$


Let $\pi: \bar{X} \rightarrow \mathbb{P}^{1}$ be the morphism given by $[x: y: z: w] \mapsto[z: w]$. Suppose that $X$ is smooth and its Picard number is at most 2 . Assume that the fiber of $\pi$ above $[1: 0]$ is singular at $[0: 0: 1: 0]$, the fiber above $[0: 1]$ has a cusp at $[0: 0: 0: 1]$, and no other fiber is cuspidal. Then we have Aut $\bar{X}=\{1\}$.

Let $L$ be the line given by $w=z=0$. The morphism $\pi$ in Proposition 3.1 is an elliptic fibration (not necessarily with a section). Each fiber is the union of the components other than $L$ in some hyperplane section through $L$. In order for $f_{1}, f_{2}$, and $f_{3}$ to satisfy all conditions of Proposition 3.1, they have to satisfy other conditions that are easier to check. The following lemma states some of these conditions. Not only are they useful for finding explicit examples, some of them will also be used in the proof of Proposition 3.1.

Lemma 3.2. Let $f_{1}, f_{2}, f_{3}, X$, and $\pi$ be as in Proposition 3.1. Then there are $f_{1}^{\prime}, f_{2}^{\prime}, f_{3}^{\prime} \in k[x, y, z, w]$ that satisfy the following conditions.

(a) $X$ is given by $w\left(x^{3}+x y^{2}+w f_{1}^{\prime}\right)+z\left(y^{3}+z f_{2}^{\prime}\right)+z w f_{3}^{\prime}=0$,

(b) $f_{1}^{\prime}, f_{2}^{\prime} \in k[x, y]$,

(c) $f_{1}^{\prime}$ is a square over $\bar{k}$,

(d) the coefficients of $z^{2}$ and $w^{2}$ in $f_{3}^{\prime}$ are nonzero,

(e) the polynomials $x^{3}+x y^{2}+w f_{1}^{\prime}$ and $y^{3}+z f_{2}^{\prime}$ are irreducible over $\bar{k}$,

(f) if $k$ is finite, say $\# k=q$, and $F$ is the $q$-th power Frobenius acting on $H_{\text {ét }}^{2}\left(\bar{X}, \mathbb{Q}_{l}\right)(1)$, and Tate's conjecture is true, then the sign of the functional equation for the characteristic polynomial of $F$ is positive.

Proof. By definition there are $f_{1}^{\prime}, f_{2}^{\prime}, f_{3}^{\prime}$ such that (a) is satisfied, namely given by $f_{i}^{\prime}=f_{i}$. After collecting all monomials of the polynomial $g=w\left(x^{3}+x y^{2}+\right.$ $\left.w f_{1}^{\prime}\right)+z\left(y^{3}+z f_{2}^{\prime}\right)+z w f_{3}^{\prime}$ that are divisible by $z w$ in the term $z w f_{3}^{\prime}$ we may assume $f_{1}^{\prime} \in k[x, y, w]$ and $f_{2}^{\prime} \in k[x, y, z]$. Since the fiber $F_{\infty}$ of $\pi$ above [1:0], given by $w=y^{3}+z f_{2}^{\prime}=0$, contains the singular point $P_{z}=[0: 0: 1: 0]$, we find that the polynomial $y^{3}+z f_{2}^{\prime}$ and its derivatives with respect to $x, y$, and $z$ all vanish at $P_{z}$. This implies that $f_{2}^{\prime}$ does not contain the variable $z$, so we have $f_{2}^{\prime} \in k[x, y]$. Similarly we find $f_{1}^{\prime} \in k[x, y]$ from the singularity $P_{w}=[0: 0: 0: 1]$ in the fiber $F_{0}$ above $[0: 1]$, which proves (b). The fact that $F_{0}$ has a cusp at $P_{w}$ is then equivalent to the fact that $f_{1}^{\prime}$ is a square in $\bar{k}[x, y]$, which proves (c). It is now easily checked that $g$ and its derivatives with respect to $x, y$, and $w$ all vanish at $P_{w}$. Since $X$ is smooth at $P_{w}$, this implies that $\partial g / \partial z$ does not vanish at $P_{w}$, which is equivalent to the coefficient of $w^{2}$ in $f_{3}^{\prime}$ being nonzero. Similarly we find from the fact that $X$ is smooth at $P_{z}$ that the coefficient of $z^{2}$ in $f_{3}^{\prime}$ is nonzero. This takes care of $(\mathrm{d})$. For $(\mathrm{e})$, note that all fibers are geometrically integral by Lemmas 2.8 and 2.9. The fibers $F_{0}$ and $F_{\infty}$ are given by $z=x^{3}+x y^{2}+w f_{1}^{\prime}=0$ and $w=y^{3}+z f_{2}^{\prime}=0$ respectively, so the polynomials $y^{3}+z f_{2}^{\prime}$ and $x^{3}+x y^{2}+w f_{1}^{\prime}$ are irreducible over $\bar{k}$. Finally, assume all hypotheses of (f). By Lemma 2.4 the Néron-Severi group $\mathrm{NS}(\bar{X})$ is generated by the class of hyperplane sections and the divisor class of the line $L$. Since $F$ acts trivially on these classes, by Proposition 2.6 and Remark 2.7 we find that there are exactly two eigenvalues of $F$ that are roots of unity, both equal to 1 . In particular, the multiplicity of the eigenvalue 1 is even, which implies that the sign of the functional equation is positive by Lemma 2.10 . 
Proof of Proposition 3.1. Replace the $f_{i}$ by the $f_{i}^{\prime}$ of Lemma 3.2. Since $X$ contains the line $L$, we find from Lemma 2.4 that $\operatorname{NS}(\bar{X})$ is generated by the class of hyperplane sections and the class $[L]$. Let $\sigma$ be any $\bar{k}$-automorphism of $\bar{X}$. By Proposition 2.3 we have $\sigma \in \operatorname{Lin} \bar{X}$ and $\sigma$ fixes $L$. The first claim implies that there exists a matrix $A$ such that $\sigma$ sends $[x: y: z: w]$ to $\left[x^{\prime}: y^{\prime}: z^{\prime}: w^{\prime}\right]$ with $\left(x^{\prime} y^{\prime} z^{\prime} w^{\prime}\right)^{t}=A \cdot(x y z w)^{t}$, where $v^{t}$ denotes the transpose of the vector $v$. Since $A$ maps the generators $z, w$ of the ideal of $L$ to generators of the same ideal, we find that $A$ is of the form

$$
\left(\begin{array}{llll}
a & b & \kappa & \lambda \\
c & d & \mu & \nu \\
0 & 0 & p & q \\
0 & 0 & r & s
\end{array}\right) .
$$

Let $g$ be the polynomial in the left-hand side of equation (2). Set $\left(x^{\prime} y^{\prime} z^{\prime} w^{\prime}\right)^{t}=$ $A \cdot(x y z w)^{t}$. As $\sigma$ is an automorphism, the polynomial $g^{\prime}=g\left(x^{\prime}, y^{\prime}, z^{\prime}, w^{\prime}\right)$ in terms of the variables $x, y, z, w$ also defines $X$, so $g^{\prime}$ is a scalar multiple of $g$. After scaling the matrix $A$ we may assume $g^{\prime}=g$. Comparing the coefficients in $g$ and $g^{\prime}$ of the monomials that are linear in $z$ and $w$ we find that the following expressions are all zero.

$$
\begin{aligned}
Q_{1} & =c^{3} p+\left(a^{3}+a c^{2}\right) r, \\
Q_{2} & =c^{3} q+\left(a^{3}+a c^{2}\right) s-1, \\
Q_{3} & =3 c^{2} d p+\left(3 a^{2} b+2 a c d+b c^{2}\right) r, \\
Q_{4} & =3 c^{2} d q+\left(3 a^{2} b+2 a c d+b c^{2}\right) s, \\
Q_{5} & =3 c d^{2} p+\left(3 a b^{2}+a d^{2}+2 b c d\right) r, \\
Q_{6} & =3 c d^{2} q+\left(3 a b^{2}+a d^{2}+2 b c d\right) s-1, \\
Q_{7} & =d^{3} p+\left(b^{3}+b d^{2}\right) r-1, \\
Q_{8} & =d^{3} q+\left(b^{3}+b d^{2}\right) s .
\end{aligned}
$$

This implies that we also have

$$
\begin{aligned}
c r & =-s d Q_{3}+r d Q_{4}+s c Q_{5}-r c Q_{6}=0, \\
c^{3} s+d^{3} r & =s d^{3} Q_{1}-r d^{3} Q_{2}-s c^{3} Q_{7}+r c^{3} Q_{8}=0 .
\end{aligned}
$$

From the first of these last two equations we find $r=0$ or $c=0$. In case of $r=0$, we find $s \neq 0$ from the fact that $A$ is invertible and from the second equation we then find $c=0$, so we have $c=0$ in either case. Since $A$ is invertible, we conclude $a \neq 0$. Then the equation $Q_{1}=0$ implies $r=0$ and $Q_{2}=0$ gives $s=a^{-3}$. From $Q_{4}=0$ it now follows that we have $3 b=0$. Equation $Q_{6}=0$ yields $s=a^{-1} d^{-2}$, which together with $s=a^{-3}$ gives $d= \pm a$. The equation $Q_{7}=0$ shows $p=d^{-3}$.

From $r=0$ we deduce that the fiber $F_{\infty}$ of $\pi$ above [1:0] is mapped to itself. All fibers are geometrically integral by Lemma 2.8 and 2.9. As integral cubic plane curves have at most one singular point (see [5], Exm. V.3.9.2), the singular point $[0: 0: 1: 0]$ of $F_{\infty}$ is unique and thus fixed by $\sigma$. This implies $\kappa=\mu=0$. The cuspidal fiber $F_{0}$ above $[0: 1]$ is sent isomorphically to the fiber above $[q: s]$. The only cuspidal fibers lie above [0:1] and possibly [1:0], so from $s \neq 0$ we find $q=0$. We deduce that $\sigma$ sends the unique cusp $[0: 0: 0: 1]$ of $F_{0}$ to itself, which implies $\lambda=\nu=q=0$. 
From Lemma 3.2 we know that $y^{3}+z f_{2}$ is irreducible, so $f_{2} \in k[x, y]$ is not divisible by $y$. Therefore the coefficient $\chi$ of $x^{2}$ in $f_{2}$ is nonzero. Comparing the coefficient of $x^{2} z^{2}$ in $g^{\prime}$ and $g$ we find from $a^{2}=d^{2}$ that we have

$$
\chi=\chi a^{2} p^{2}=\chi a^{2} d^{-6}=\chi a^{-4},
$$

which implies that we have $a^{4}=1$. By Lemma 3.2 the coefficient of $w z^{3}$ in $g$ is also nonzero. Comparing this to the coefficient of $w z^{3}$ in $g^{\prime}$ we now find $1=d^{-9} a^{-3}=$ $d^{-1} a$, so $a=d$. After dividing $A$ by $a$ we may assume $1=a=d=p=s$. By comparing the coefficient of $x y z^{2}$ in $g$ and $g^{\prime}$ we then get $2 \chi b=0$, so $2 b=0$. Together with $3 b=0$ this gives $b=0$ and we find that $\sigma$ is the identity. As this holds for every $\sigma \in$ Aut $\bar{X}$ we find Aut $\bar{X}=\{1\}$.

Remark 3.3. The main idea of the proof of Proposition 3.1 is that the line $L$ given by $z=w=0$ is fixed by any automorphism. This implies that any automorphism permutes the fibers of $\pi$. We picked some extra conditions to ensure that some of these fibers have to be fixed, which yields enough information to deduce that every automorphism is trivial. These conditions are by no means general. They are carefully chosen such that on one hand they are easy enough to verify the proof without a computer, while on the other hand it leaves ample examples among which to search for surfaces with the right Picard number.

Before we give an explicit example that satisfies all conditions of Proposition 3.1, we will sketch the odds we had to beat to find one. Based on Lemma 3.2, the least number of monomials of an equation as in Proposition 3.1 that satisfies all conditions of the proposition is 7. This minimum is achieved if and only if there are $\alpha, \beta, \gamma, \delta \in k^{*}$, such that the quartic surface $X$ is given by

$$
w\left(x^{3}+x y^{2}+\alpha w y^{2}\right)+z\left(y^{3}+\beta z x^{2}\right)+z w\left(\gamma z^{2}+\delta w^{2}\right)=0 .
$$

The map $[x: y: z: w] \mapsto[-x:-y: z: w]$ gives an isomorphism between the surfaces corresponding to $(\alpha, \beta, \gamma, \delta)$ and $(-\alpha,-\beta,-\gamma,-\delta)$, while the map $[x: y: z$ : $w] \mapsto[x:-y:-z: w]$ gives an isomorphism between the surfaces corresponding to $(\alpha, \beta, \gamma, \delta)$ and $(\alpha, \beta,-\gamma,-\delta)$. For $k=\mathbb{F}_{3}$ this gives four quadruples of isomorphic surfaces. The four nonisomorphic surfaces are all smooth. For one of them the fibration $\pi$ has reducible components, so its Picard number is at least 3 by Lemma 2.9. For the remaining three surfaces $X$ we computed the characteristic polynomial of Frobenius on $H_{\text {ét }}^{2}\left(\bar{X}, \mathbb{Q}_{l}\right)(1)$. The sign of the functional equation of one of them is negative, which gives a bound for the Picard number larger than 2 by Lemma 3.2. Unfortunately, Proposition 2.6 gives an upper bound of at least 4 for the remaining two Picard numbers as well.

We therefore look at surfaces given by (3) with an extra term $\epsilon y z^{2} w$ for some $\epsilon \in \mathbb{F}_{3}^{*}$. In this case we get eight nonisomorphic smooth surfaces, one of which has a hyperplane section that contains three components. For only one of the remaining seven surfaces the sign of the corresponding functional equation is positive. We computed the characteristic polynomial of Frobenius in that case and found from Proposition 2.6 that the Picard number is at most 2 . This yields the following corollary.

Corollary 3.4. Let $k$ be any field of characteristic 0 or 3 . Let $X$ in $\mathbb{P}^{3}$ over $k$ be given by

$$
x^{3} w-x^{2} z^{2}+x y^{2} w+y^{3} z+y^{2} w^{2}+y z^{2} w-z^{3} w+z w^{3}=0
$$


Then we have Aut $\bar{X}=\{1\}$.

Proof. Let $g$ denote the polynomial in the given equation. Note that the equation is of the form used in Proposition 3.1 with $f_{1}=y^{2}, f_{2}=-x^{2}$, and $f_{3}=y z-z^{2}+w^{2}$. Although without a computer algebra package it is a bit of work to show that $X$ is smooth, with such a package one easily checks that this is the case. Let $X_{0}$ and $X_{3}$ denote the surfaces defined by $g=0$ over $\mathbb{Q}$ and $\mathbb{F}_{3}$ respectively. We will show $\operatorname{rank} \mathrm{NS}\left(\bar{X}_{3}\right) \leq 2$. Each of the nonsingular fibers of the fibration $\pi$ as in Proposition 3.1 can be given the structure of an elliptic curve by searching for a rational point on it. There are fast algorithms to compute the number of points on elliptic curves, implemented in for instance magma. Summing over all fibers, we find the number of points on $X_{3}$ over $\mathbb{F}_{3^{n}}$ for $n \in\{1, \ldots, 10\}$. These numbers are

$$
17,101,812,6545,58502,531902,4788164,43074713,387494444,3486985076 \text {. }
$$

As in [14], Prop. 7.1, and [15], Thm. 3.1, we can use the Lefschetz formula to find the characteristic polynomial of Frobenius acting on $H_{\text {ét }}^{2}\left(\bar{X}_{3}, \mathbb{Q}_{l}\right)(1)$ from these numbers. Here we use the fact that we already know a Galois invariant subspace of $H_{\text {ét }}^{2}\left(\bar{X}_{3}, \mathbb{Q}_{l}\right)(1)$, generated by a hyperplane section and the line $L$. In $\mathbb{Q}[t]$ the characteristic polynomial factors into irreducible factors as

$$
\begin{aligned}
\frac{1}{3}(t-1)^{2}\left(3 t^{20}\right. & -t^{19}-t^{17}+2 t^{16}+2 t^{15}-t^{13}-t^{12}+t^{11} \\
& \left.+t^{10}+t^{9}-t^{8}-t^{7}+2 t^{5}+2 t^{4}-t^{3}-t+3\right) .
\end{aligned}
$$

The roots of the irreducible factor of degree 20 are not integral, so they are not roots of unity. By Proposition 2.6 we find $\operatorname{rank} \mathrm{NS}\left(\bar{X}_{3}\right) \leq 2$. From Proposition 2.5 we also find $\operatorname{rank} \operatorname{NS}\left(\bar{X}_{0}\right) \leq 2$. Depending on the characteristic we have either $X=X_{3} \times k$ or $X=X_{0} \times k$. As the Néron-Severi group is algebraic, we conclude $\operatorname{NS}(\bar{X})=\operatorname{NS}\left(\bar{X}_{3}\right)$ or $\operatorname{NS}(\bar{X})=\operatorname{NS}\left(\bar{X}_{0}\right)$. In both cases we conclude $\operatorname{rank} \operatorname{NS}(\bar{X}) \leq 2$. The fibers of $\pi$ above $[0: 1]$ and $[1: 0]$ have a cusp at the points $[0: 0: 0: 1]$ and $[0: 0: 1: 0]$ respectively. In characteristic 0 there are 20 more singular fibers. By Lemma 2.9 these fibers are all nodal curves. In characteristic 3 there are 14 more singular fibers. A tedious calculation shows that again none of the corresponding fibers has a cusp. From Proposition 3.1 we deduce Aut $\bar{X}=\{1\}$.

\section{Explicit examples containing a conic}

Proposition 4.1. Let $k$ be any field with elements $\alpha, \beta$ satisfying $\alpha^{3} \beta \neq \alpha \beta^{3}$. Let $f \in k[x, y, z, w]$ be a homogeneous polynomial of degree 3 , such that the coefficients of $y^{3}$ and $z^{3}$ in $f$ are different, or the coefficients of $x^{2} y$ and $x^{2} z$ in $f$ are different. Suppose that the surface $X$ in $\mathbb{P}^{3}$ given by

$$
w f=(x y+x z+\alpha y z)(x y+x z+\beta y z)
$$

is smooth with Picard number at most 2. Then we have Aut $\bar{X}=\{1\}$.

As in the previous section, the following lemma will be useful both for the proof of this proposition and for constructing examples that satisfy all conditions.

Lemma 4.2. Let $f$ be as in Proposition 4.1. Then the following conditions hold.

(a) The coefficients of $x^{3}, y^{3}$, and $z^{3}$ in $f$ are nonzero. 
(b) If $k$ is finite, say $\# k=q$, and $F$ is the $q$-th power Frobenius acting on $H_{\text {ét }}^{2}\left(\bar{X}, \mathbb{Q}_{l}\right)(1)$, and Tate's conjecture is true, then the sign of the functional equation for the characteristic polynomial of $F$ is positive.

(c) Suppose $k^{\prime}$ is a finite quadratic subfield of $k$, say $\# k^{\prime}=q$, the elements $\alpha$ and $\beta$ are conjugate over $k^{\prime}$, and $f \in k^{\prime}[x, y, z, w]$. Then $X$ is defined over $k^{\prime}$. If $F$ is the q-th power Frobenius acting on $H_{\text {ét }}^{2}\left(\bar{X}, \mathbb{Q}_{l}\right)(1)$, and Tate's conjecture is true, then the sign of the functional equation for the characteristic polynomial of $F$ is negative.

Proof. Note that $X$ contains the points $P_{1}=[1: 0: 0: 0], P_{2}=[0: 1: 0: 0]$, and $P_{3}=[0: 0: 1: 0]$. One easily checks that for $i=1,2,3$, the surface $X$ is smooth at $P_{i}$ if and only if we have $f\left(P_{i}\right) \neq 0$. This implies (a). For (b) and (c), note that by Lemma 2.4 the Néron-Severi group $\mathrm{NS}(\bar{X})$ is generated by the class $H$ of hyperplane sections and the divisor class of the conic $C$ given by $w=x y+x z+\alpha y z=0$. In case (b), $F$ acts trivially on these classes, and the proof proceeds as the proof of (f) of Lemma 3.2. In case (c), $F$ acts trivially on $H$, and by a nontrivial quadratic character on the class $2[C]-H$. By Proposition 2.6 and Remark 2.7 this implies that counted with multiplicity, there are exactly two eigenvalues of $F$ that are roots of unity, equal to 1 and -1 respectively. In particular, the multiplicity of the eigenvalue 1 is odd, which implies that the sign of the functional equation is negative by Lemma 2.10.

Proof of Proposition 4.1. Let $C$ and $\tilde{C}$ denote the conics given by $w=x y+$ $x z+\alpha y z=0$ and $w=x y+x z+\beta y z=0$ respectively. As both are contained in $X$, we find from Lemma 2.4 that $\operatorname{NS}(\bar{X})$ is generated by the class of hyperplane sections and the class $[C]$. Let $\sigma$ be a $\bar{k}$-automorphism of $\bar{X}$. From Proposition 2.3 we conclude that we have $\sigma \in \operatorname{Lin} \bar{X}$ and that $\sigma$ fixes the plane containing $C$, which also contains $\tilde{C}$. It follows that $\sigma$ permutes the intersection points of $C$ and $\tilde{C}$. These are $P_{x}=[1: 0: 0: 0], P_{y}=[0: 1: 0: 0]$, and $P_{z}=[0: 0: 1: 0]$, where the first has multiplicity 2 and the others have multiplicity 1 . This implies that $\sigma$ fixes $P_{x}$ and either it also fixes $P_{y}$ and $P_{z}$, or it switches these two. Therefore $\sigma$ is given by $[x: y: z: w] \mapsto\left[x^{\prime}: y^{\prime}: z^{\prime}: w^{\prime}\right]$ with $\left(x^{\prime} y^{\prime} z^{\prime} w^{\prime}\right)^{t}=A(x y z w)^{t}$ for a matrix $A$ of the form

$$
\left(\begin{array}{cccc}
p & 0 & 0 & \lambda \\
0 & q & 0 & \mu \\
0 & 0 & r & \nu \\
0 & 0 & 0 & s
\end{array}\right), \quad \text { or } \quad\left(\begin{array}{cccc}
p & 0 & 0 & \lambda \\
0 & 0 & r & \mu \\
0 & q & 0 & \nu \\
0 & 0 & 0 & s
\end{array}\right) \text {, }
$$

with $p, q, r, s \in \bar{k}^{*}$ and $\lambda, \mu, \nu \in \bar{k}$. Let $g$ denote the polynomial $w f-(x y+x z+$ $\alpha y z)(x y+x z+\beta y z)$.

Suppose we are in the first (diagonal) case. Then with $x^{\prime}=p x+\lambda w, y^{\prime}=q y+\mu w$, $z^{\prime}=r z+\nu w$, and $w^{\prime}=s w$ the polynomial $g^{\prime}=g\left(x^{\prime}, y^{\prime}, z^{\prime}, w^{\prime}\right)$ in terms of the variables $x, y, z$, and $w$ also defines $\bar{X}$, so it is a constant multiple of the polynomial $g$. After scaling $A$ we may assume $g^{\prime}=g$. For any monomial $M$, let $c_{M}$ and $c_{M}^{\prime}$ denote the coefficients of $M$ in $g$ and $g^{\prime}$ respectively. Then for every monomial $M$ we have $c_{M}=c_{M}^{\prime}$. From the assumption $\alpha^{3} \beta \neq \alpha \beta^{3}$ we find $\alpha+\beta \neq 0$, so $c_{M} \neq 0$ for $M \in S_{1}=\left\{x y^{2} z, x y z^{2}, x^{2} y^{2}\right\}$. As the coefficient of $x^{3}$ in $f$ is nonzero by Lemma 4.2, we also conclude $c_{w x^{3}} \neq 0$. Note that the terms $(x y+x z+\alpha y z)(x y+x z+\beta y z)$ and $\left(x^{\prime} y^{\prime}+x^{\prime} z^{\prime}+\alpha y^{\prime} z^{\prime}\right)\left(x^{\prime} y^{\prime}+x^{\prime} z^{\prime}+\beta y^{\prime} z^{\prime}\right)$ do not contribute to $c_{M}$ and $c_{M}^{\prime}$ for 
$M=w x^{3}$. Therefore the equations $c_{M}^{\prime}=c_{M}$ for $M \in S_{1} \cup\left\{w x^{3}\right\}$ give $s p^{3}=p q^{2} r=$ $p q r^{2}=p^{2} q^{2}=1$, which implies $p=q=r=s$. After scaling $A$ we may assume $p=q=r=s=1$. Then for $M \in\left\{x y^{2} w, x z^{2} w, y z^{2} w\right\}$ the equations $c_{M}=c_{M}^{\prime}$ give

$$
\begin{aligned}
-2 \lambda-(\alpha+\beta) \nu+c_{x y^{2} w} & =c_{x y^{2} w}, \\
-2 \lambda-(\alpha+\beta) \mu+c_{x z^{2} w} & =c_{x z^{2} w}, \\
-(\alpha+\beta) \lambda-2 \alpha \beta \mu+c_{y z^{2} w} & =c_{y z^{2} w} .
\end{aligned}
$$

By hypothesis we have $\alpha^{2} \neq \beta^{2}$. One easily checks that this implies that this system of linear equations has no nontrivial solutions in $\lambda, \mu$, and $\nu$, so we have $\lambda=\mu=\nu=0$ and $\sigma$ is the identity.

Suppose we are in the second (nondiagonal) case. Now we have $x^{\prime}=p x+\lambda w$, $y^{\prime}=r z+\mu w, z^{\prime}=q y+\nu w$, and $w^{\prime}=s w$. As before we may assume that $g^{\prime}=$ $g\left(x^{\prime}, y^{\prime}, z^{\prime}, w^{\prime}\right)$ equals $g$, and we can again compare the coefficients $c_{M}$ and $c_{M}^{\prime}$ of the monomials $M$ in $g$ and $g^{\prime}$ respectively. As in the first case we find $p=q=r=s$, so we can scale $A$ to get $p=q=r=s=1$. This gives $c_{w z^{3}}^{\prime}=c_{w y^{3}}$. From $c_{w z^{3}}^{\prime}=c_{w z^{3}}$ we then deduce $c_{w y^{3}}=c_{w z^{3}}$, so the coefficients of $y^{3}$ and $z^{3}$ in $f$ are equal. For $M \in\left\{x^{2} y w, x^{2} z w\right\}$ the equations $c_{M}=c_{M}^{\prime}$ give

$$
\begin{aligned}
& -2 \mu-2 \nu+c_{x^{2} z w}=c_{x^{2} y w}, \\
& -2 \mu-2 \nu+c_{x^{2} y w}=c_{x^{2} z w},
\end{aligned}
$$

which has no solution in any characteristic unless $c_{x^{2} y w}=c_{x^{2}} z w$. This implies that also the coefficients of $x^{2} z$ and $x^{2} y$ in $f$ are equal, which contradicts our assumptions. We conclude that this case does not occur, so $\sigma$ is the identity. As this holds for every $\sigma \in \operatorname{Aut} \bar{X}$ we find Aut $\bar{X}=\{1\}$.

Remark 4.3. As for Proposition 3.1, the conditions in Proposition 4.1 are by no means general.

Remark 4.4. We will give examples of quartic surfaces $X$ for which Proposition 4.1 immediately tells us that we have Aut $\bar{X}=\{1\}$. To do this over a field $k$ we need $\alpha, \beta \in \bar{k}$, such that $\alpha \beta(\beta-\alpha)(\beta+\alpha) \neq 0$ and the right-hand side of the equation in Proposition 4.1 is defined over $k$. If $k$ has at least 4 elements than the existence of such $\alpha$ and $\beta$ follows from the fact that for any nonzero $\alpha$ the polynomial $\alpha X\left(X^{2}-\alpha^{2}\right)$ has at most 3 roots. For $k=\mathbb{F}_{2}$ we can take $(\alpha, \beta)=(\zeta, \zeta+1)$ for some $\zeta \in \mathbb{F}_{4}$ with $\zeta^{2}+\zeta+1=0$. For $k=\mathbb{F}_{3}$ we can take $(\alpha, \beta)=(1+i, 1-i)$ for some element $i \in \mathbb{F}_{9}$ with $i^{2}=-1$.

Corollary 4.5. Let $k$ be any field of characteristic 0 or 2 and let $X \subset \mathbb{P}^{3}$ over $k$ be given by

$$
w\left(x^{3}+y^{3}+z^{3}+x^{2} z+x w^{2}\right)=x^{2} y^{2}+2 x^{2} y z+x^{2} z^{2}-x y^{2} z-x y z^{2}+y^{2} z^{2} .
$$

Then we have Aut $\bar{X}=\{1\}$.

Proof. Let $X_{0}$ and $X_{2}$ be the surfaces defined by the given equation over $\mathbb{Q}$ and $\mathbb{F}_{2}$ respectively. Although it is tedious work without a computer algebra package, one easily checks that $X_{0}$ and $X_{2}$ are smooth. That means that $p=2$ is a prime of good reduction for $X_{0}$. In [15], Rem. 6 , it was shown that we have rank NS $\left(\bar{X}_{2}\right) \leq 2$. From Proposition 2.5 we also find $\operatorname{rank} \operatorname{NS}\left(\bar{X}_{0}\right) \leq 2$. Because the Néron-Severi group is 
algebraic, we find as in the proof of Corollary 3.4 that we have $\operatorname{rank} \operatorname{NS}(\bar{X}) \leq 2$. Let $\zeta$ be an element (possibly in a quadratic extension of $k$ ) that satisfies $\zeta^{2}+\zeta+1=0$. Then the equation for $X$ is of the form given in Proposition 4.1 with

$$
(\alpha, \beta, f)=\left(\zeta, \zeta^{2}, x^{3}+y^{3}+z^{3}+x^{2} z+x w^{2}\right) .
$$

From Proposition 4.1 we find Aut $\bar{X}=\{1\}$.

\section{Proof of the main theorems}

For characteristics 0,2 , and 3 we have seen examples of quartic surfaces with trivial automorphism group in Corollary 3.4 and 4.5. Kiran Kedlaya has also found examples of triples $(\alpha, \beta, f)$ in characteristics $5, \ldots, 19$ that satisfy all conditions of Proposition 4.1 .

Proposition 5.1. Take $\alpha=1, \beta=3$, and $(p, f)$ one of the following pairs.

\begin{tabular}{|c|c|}
\hline$p$ & $f$ \\
\hline 5 & $3 x^{3}+3 x y^{2}-x y w+3 x z w-x w^{2}+y^{3}-y^{2} w+2 z^{3}+w^{3}$ \\
7 & $-2 x^{3}+2 x^{2} y+2 x^{2} w+y^{3}+3 y^{2} w+y z w-y w^{2}+2 z^{3}$ \\
11 & $-5 x^{3}-2 x^{2} y-5 x y^{2}-2 x z^{2}+y^{3}-y z^{2}+2 z^{3}-4 w^{3}$ \\
13 & $3 x^{3}-6 x^{2} z+y^{3}-6 y w^{2}+2 z^{3}$ \\
17 & $-x^{3}+8 x^{2} y-x y w+y^{3}-y^{2} w+2 z^{3}+5 z^{2} w-5 z w^{2}$ \\
19 & $6 x^{3}+3 x^{2} z+7 x y w-7 x z^{2}+8 x z w-9 x w^{2}+y^{3}+$ \\
& $-y^{2} w-5 y z^{2}+5 y w^{2}+2 z^{3}-4 z^{2} w-2 z w^{2}$ \\
\hline
\end{tabular}

Then for every field $k$ of characteristic $p$, all conditions of Proposition 4.1 are satisfied for the triple $(\alpha, \beta, f)$.

Proof. The bound of the Picard number is the only condition that is not trivial to check, see [7].

Proof of Theorem 1.5. Corollary 4.5 gives an explicit example in characteristics 0 and 2. For characteristic 3 an example is given by Corollary 3.4. For characteristics $5, \ldots, 19$ we use Proposition 4.1 with $\alpha=1, \beta=3$, and $(p, f)$ as in Proposition 5.1.

Proof of Theorem 1.6. Let $f$ denote the polynomial in the given equation and set $f_{1}=\frac{1}{2}(x+y)^{2}, f_{2}=x^{2}+3 x y+3 y^{2}$, and $f_{3}=x^{2}+\frac{1}{2} x z+5 y z+\frac{1}{2} z^{2}+\frac{7}{2} z w-2 w^{2}$. Then we have $\frac{1}{2} f=w\left(x^{3}+x y^{2}+w f_{1}\right)+z\left(y^{3}+z f_{2}\right)+z w f_{3}$, so $X$ is as in Proposition 3.1. One easily checks that $X$ is smooth with good reduction at 3 . Let $X_{3}$ denote the reduction at 3 . For $n=1, \ldots, 10$, we compute the number of points on $X_{3}$ over $\mathbb{F}_{3^{n}}$. These numbers are

$$
\text { 15, 107, 639, 6935, 59790, 533729, 4790661, 43039079, 387592263, 3486831422. }
$$

As in the proof of Corollary 3.4, and as in [14], Prop. 7.1, and [15], Thm. 3.1, we can find the characteristic polynomial of Frobenius acting on $H_{\text {ét }}^{2}\left(\bar{X}_{3}, \mathbb{Q}_{l}\right)(1)$ from these numbers. In $\mathbb{Q}[x]$ the characteristic polynomial factors into irreducible factors as

$$
\begin{aligned}
\frac{1}{3}(x-1)^{2}\left(3 x^{20}\right. & +x^{19}-x^{18}+5 x^{17}-3 x^{15}+4 x^{14}-2 x^{13}-2 x^{12}+x^{11} \\
& \left.-3 x^{10}+x^{9}-2 x^{8}-2 x^{7}+4 x^{6}-3 x^{5}+5 x^{3}-x^{2}+x+3\right) .
\end{aligned}
$$


As the roots of the factor of degree 20 are not integral, we find rank $\operatorname{NS}\left(\bar{X}_{3}\right) \leq 2$ from Proposition 2.6. Then from Proposition 2.5 we also find $\operatorname{rank} \mathrm{NS}(\bar{X}) \leq 2$. As before, let $\pi$ denote the elliptic fibration given by $[x: y: z: w] \mapsto[z: w]$. The fiber $F_{\infty}$ above $[1: 0]$ has a node at $[0: 0: 1: 0]$. The fiber $F_{0}$ above $[0: 1]$ has a cusp at $[0: 0: 0: 1]$. There are 21 more singular fibers, all conjugate over $\mathbb{Q}$. By Lemma 2.9 these are all nodal curves. We conclude that all conditions of Proposition 3.1 are fulfilled, so we find Aut $\bar{X}=\{1\}$.

Consider the curve $C=X \cap H$, where $H$ is the hyperplane given by $w+x=z$. Note that $C$ can be parametrized by $\tau: \mathbb{P}^{1} \rightarrow C,[s: 1] \mapsto[z-w: y: z: w]$ with

$$
y=-8 s^{4}-4 s^{3}+6 s^{2}-1, \quad z=2 s^{2}\left(4 s^{2}+2 s-1\right), \quad w=2 s .
$$

The curve $C$ intersects each fiber $F$ of $\pi$ with multiplicity $\operatorname{deg} F=3$, so the restriction of $\pi$ to $C$ has degree 3. Identify the function field $k(C)$ of $C$ with $k(s)$ through $\tau$. Identify the function field of the base curve $\mathbb{P}^{1}$ of $\pi$ with $k(t)$. The composition $\pi \tau$ is given by $[s: 1] \mapsto\left[s\left(4 s^{2}+2 s-1\right): 1\right]$, so the corresponding field extension $k(s) / k(t)$ of degree 3 is given by $t=s\left(4 s^{2}+2 s-1\right)$. The map $\tau$ corresponds to a point $\mathcal{O}$, defined over $k(s)$, on the generic fiber $E$ of $\pi$, giving $E$ the structure of an elliptic curve over $k(s)$. The sum $S$ of the two conjugates of $\mathcal{O}$, both defined over $\overline{k(t)}$, is also defined over $k(s)$. The map $\pi \tau: \mathbb{P}^{1}(s) \rightarrow \mathbb{P}^{1}(t)$ is ramified at $s_{0}=\frac{1}{6}$ above $t_{0}=-\frac{5}{54}$, where by abuse of notation we denote the point $[\alpha: \beta]$ by $\alpha \beta^{-1}$. The third point above $t_{0}$ is $s_{1}=-\frac{5}{6}$. Specializing at $s=s_{1}$ we find that $E$ specializes to the elliptic fiber $F_{t_{0}}$ above $t_{0}$, the point $\mathcal{O}$ specializes to $\mathcal{O}_{s_{1}}=\tau\left(s_{1}\right)=[295: 263: 25:-270]$, and the point $S$ specializes to $S_{s_{1}}=2 Q$ for $Q=\tau\left(s_{0}\right)=[59: 139: 5:-54]$. With these explicit equations, a standard computation shows that $Q$, and therefore $S_{s_{1}}$, has infinite order on the elliptic curve $F_{t_{0}}$ with $\mathcal{O}_{s_{1}}$ as origin. This implies that $S$ has infinite order on the generic fiber $E$. The infinitely many multiples of $S$ correspond to infinitely many rational curves on $X$, all with infinitely many rational points, obtained from specializing $s$. This shows that the set $X(\mathbb{Q})$ of rational points is Zariski dense in $X$.

Remark 5.2. In the notation of [1], the curve $C$ in the proof of Theorem 1.6 is a rational $n t$-multisection of $\pi$. This follows from the proof. A highbrow argument for this fact is that $C$ is saliently ramified, because the restriction of $\pi$ to $C$ ramifies in the smooth fiber above $t=-\frac{5}{54}$, see [1], Prop. 4.4.

To find a surface with such a multisection, we fixed a hyperplane $H$, three points $P_{1}, P_{2}$, and $P_{3}$, and searched for $f_{1}, f_{2}$, and $f_{3}$ such that for $X$ as in Proposition 3.1 the intersection $C=X \cap H$ is singular at the points $P_{1}, P_{2}$, and $P_{3}$. As the arithmetic genus of any hyperplane section is $\frac{1}{2}(4-1)(4-2)=3$, this ensures that $C$ has geometric genus 0, see [5], Exm. V.3.9.2. Since $C$ intersects the line $L$ given by $w=z=0$ in a smooth point, we can parametrize $C$. Per point $P_{i}$ we get three equations for the indeterminate coefficients of the $f_{j}$. If the $P_{i}$ are collinear, then $X \cap H$ will be the union of a line and a cubic curve, which means that the Picard number of $X$ will be more than 2. We therefore pick the $P_{i}$ such that modulo 3 they are not collinear. Setting $f_{1}=a(y+b x)^{2}, f_{2}=c x^{2}+d x y+e y^{2}$ and $f_{3}=\sum_{M} c_{M} M$, where $M$ runs over all 10 monomials of degree 2 in the variables $x, y, z, w$, we find 9 equations in 15 variables. Choosing $H$ to be given by $w+x=z$, and $[1: 1: 0:-1]$, $[-1:-1: 1: 2]$, and $[1:-1: 1: 0]$ for the $P_{i}$, the equations reduce to 9 linearly 
independent linear equations, so modulo 3 we find $3^{15-9}=729$ candidate surfaces. Out of all these surfaces, only 157 are nonsingular. For 64 of these, the morphism $\pi$ has a reducible fiber, making the Picard number more than 2. Out of the remaining 93 surfaces, 70 have a reducible hyperplane section defined over $\mathbb{F}_{9}$ that does not contain $L$, which also implies larger Picard number than 2. For the last 23 surfaces we computed the first 10 traces of powers of Frobenius. For ten of these surfaces the sign of the functional equation could be determined to be negative, so the bound for the Picard number is larger than 2 by Lemma 3.2. For eight surfaces the sign could not be determined yet. For four of these, either sign would give an upper bound of at least 4. Determining the sign for the other four requires substantial extra computing time. For the last five surfaces we could determine the sign to be positive. For only one of these, the upper bound for the Picard number is 2. This is the surface of Theorem 1.6.

Proof of Theorem 1.7. The given surface reduces modulo 5 to the surface of Proposition 4.1 for $\alpha=1, \beta=3$, and $f$ as in Proposition 5.1 for $p=5$. From that proposition we find that $X$ has good reduction at 5 and since the reduction has Picard number 2, we also find $\operatorname{rank} \operatorname{NS}(\bar{X}) \leq 2$ from Proposition 2.5. All conditions of Proposition 4.1 are fulfilled, so we find Aut $\bar{X}=\{1\}$. By Proposition 2.4 the NéronSeveri group is generated by the class $H$ of hyperplane sections and the class of the conic $C$ given by $w=x y+x z+y z=0$, with $H^{2}=4, C^{2}=-2$, and $H \cdot C=2$. For any nonzero class $D=a H+b[C]$ we find $D^{2}=(2 a+b)^{2}-3 b^{2}$, so $D^{2} \neq 0$. Because a fiber of any fibration of $X$ has self intersection 0 , we conclude that there is no such fibration. The conic $C$ has a point $[1: 0: 0: 0]$, so it contains infinitely many rational points.

\section{Acknowledgements}

The author thanks Bjorn Poonen for bringing this problem to his attention, Kiran Kedlaya for computing various characteristic polynomials, and CRM at Montréal for the hospitality and support during the time in which this paper was written.

\section{References}

[1] F. Bogomolov and Yu. Tschinkel,Density of rational points on elliptic K3 surfaces. Asian J. Math. 4 (2000), no. 2, 351-368.

[2] H. C. Chang, On plane algebraic curves. Chinese J. Math. 6 (1978), no. 2, 185-189.

[3] R. Friedman, Algebraic surfaces and holomorphic vector bundles. Universitext, Springer 1998.

[4] W. Fulton, Intersection theory, second edition. Springer 1998 .

[5] R. Hartshorne, Algebraic geometry. Graduate Texts in Mathematics, No. 52. Springer-Verlag, New York-Heidelberg, 1977.

[6] K. Kedlaya, Counting points on hyperelliptic curves using Monsky-Washnitzer cohomology. J. Ramanujan Math. Soc. 16 (2001), no. 4, 323-338.

[7] _ Bounding Picard numbers of surfaces using p-adic cohomology. arXiv: math. NT/0601508

[8] H. Matsumura and P. Monsky, On the automorphisms of hypersurfaces. J. Math. Kyoto Univ. 3 (1963) 347-361.

[9] N. Nygaard, and A. Ogus, Tate's conjecture for K3 surfaces of finite height. Ann. of Math. 122 (1985) 461-507.

[10] B. Poonen, Varieties without extra automorphisms III: hypersurfaces. Finite Fields Appl. 11 (2005) 230-268. 
[11] J. H. Silverman, Advanced topics in the arithmetic of elliptic curves. Graduate Texts in Mathematics, 151. Springer-Verlag, New York, 1994.

[12] J. Tate, Algebraic cycles and poles of zeta functions. 1965 Arithmetical Algebraic Geometry (Proc. Conf. Purdue Univ., 1963) pp. 93-110.

[13] B. Van Geemen, Some remarks on Brauer groups of K3 surfaces. Adv. Math. 197 (2005) 222247.

[14] R. Van Luijk, An elliptic K3 surface associated to Heron triangles. arXiv:math.AG/0411606

[15] K3 surfaces with Picard number one and infinitely many rational points. arXiv:math.AG/0506416

Department of Mathematics, University of California, Berkeley, CA 94720

E-mail address: rmluijk@gmail.com 